\title{
THE EFFECT OF SIZE OF ARTICULATION SPACE ON THE EMERGENCE OF COMBINATORIAL STRUCTURE
}

\author{
HANNAH LITTLE, BART DE BOER \\ Artificial Intelligence Laboratory, Vrije Universiteit Brussel, Pleinlaan 2 \\ 1050 Brussels, Belgium \\ hannah@ai.vub.ac.be,bart@arti.vub.ac.be
}

The emergence of structure on a phonological level has been hypothesised to be the result of pressures imposed when the number of meanings increases, as language needs a more efficient way to create new word forms. However, recent evidence from newly emerging sign-languages such as Al-Sayyid Bedouin Sign Language suggest that languages can have thousands of words without a level of phonological patterning (Sandler, Aronoff, Meir, \& Padden, 2011). Del Giudice (2012) hypothesises that the lack of phonological patterning in emerging sign languages could be because the signal space in sign languages is much bigger than in spoken languages, and this allows for a greater number of distinct signals without the need for combinatoriality, but this hypothesis is yet to be tested experimentally.

The current study falls into a tradition of work looking at linguistic structure emerging in laboratory experiments using human participants. Language displays structure on two levels, on a compositional (morphosyntactic) level, as well as on a combinatorial (phonological) level. This is known as "duality of patterning". Most previous experimental work has looked at structure emerging on a compositional level. However, a small amount of work has started exploring the emergence of structure on a combinatorial level, most notably the work of Verhoef (2012), which used signals created by slide whistles in an iterated learning paradigm. Participants learned a set of 12 whistled signals before reproducing them from memory. Participants' reproductions became the input for the next participant. Combinatorial structure was shown to emerge over generations. Whistled signals are ideal for the purposes of investigating the emergence of speech as they use a continuous articulatory space, but limit interference from participants existing linguistic knowledge.

The current study builds on Verhoef's (2012) experiment by running a new condition, which restricts the articulation space of the slide whistle with a stopper, as well as an unrestricted condition. Participants in the first generation of both conditions heard an initial set of signals created within the restricted articulation range so as to make the chains in both conditions directly comparable, as they had 
the same initial input, as well as allowing us to see whether and how participants explored a larger articulation space than that used by their input signals.

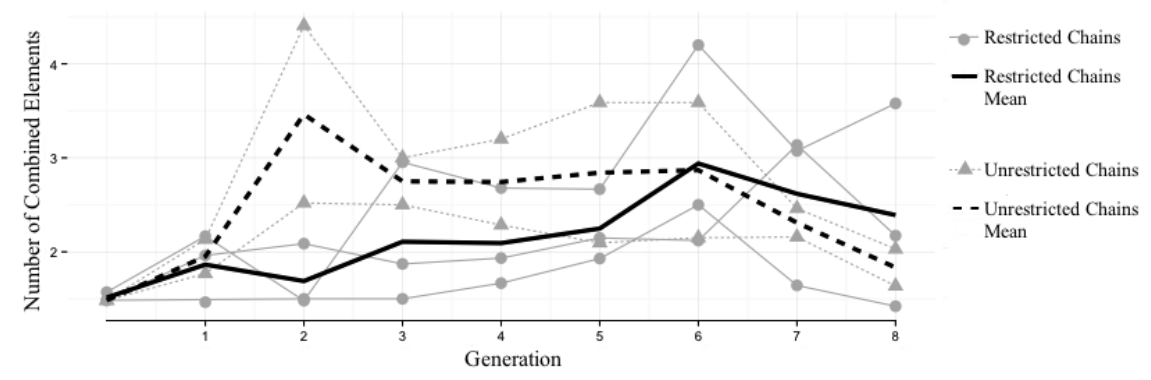

Figure 1. Number of element combinations repeated in participants' output within and across chains. Both conditions show initial emergence of combinatoriality, but only in the restricted condition does the generation predict the level of combinatoriality $R^{2}=.14, F_{1,22}=4.728, p<.05$.

The results show that in the restricted condition there is a more consistent growth of combinatorial structure (Figure 1.). Data will also be presented on the rates of reinvention after features have been lost from a chain, as well as looking at how transmission error and learnability are affected by condition.

The field of the evolution of speech is currently divided between work dealing with the emergence of the physiological capacity for speech (phonetic) or the cognitive capacity for speech (phonological). Some researchers do not even regard phonological evolution as part of speech evolution at all, rather a problem in the wider field of the evolution of language. However, the current study shows that it is important to consider both phonological and phonetic evolution when considering the emergence of combinatorial structure, as physiological factors (the size of the articulation space) can affect how structure emerges on a phonological level, demonstrating that combinatorial structure is not only subject to the cognitive biases demonstrated by Verhoef (2012).

\section{References}

Del Giudice, A. (2012). The emergence of duality of patterning through iterated learning: Precursors to phonology in a visual lexicon. Language and cognition, 4(4), 381-418.

Sandler, W., Aronoff, M., Meir, I., \& Padden, C. (2011). The gradual emergence of phonological form in a new language. Natural language \& linguistic theory, 29(2), 503-543.

Verhoef, V. (2012). The origins of duality of patterning in artificial whistled languages. Language and cognition, 4(4), 357-380. 